\title{
The Asymptotic Behavior for a Regularized Model of 3D Nonlinear-Viscous Fluid with Delay
}

\author{
Dan Yi, Chaosheng Zhu* \\ School of Mathematics and Statistics, Southwest University, Chongqing, China \\ Email: 784968541@qq.com, *zcs@swu.edu.cn
}

How to cite this paper: Yi, D. and Zhu, C.S. (2019) The Asymptotic Behavior for a Regularized Model of 3D Nonlinear-Viscous Fluid with Delay. Open Access Library Journal, 6: e5908.

https://doi.org/10.4236/oalib.1105908

Received: November 8, 2019

Accepted: November 25, 2019

Published: November 28, 2019

Copyright () 2019 by author(s) and Open Access Library Inc.

This work is licensed under the Creative Commons Attribution International License (CC BY 4.0).

http://creativecommons.org/licenses/by/4.0/

\begin{abstract}
In this paper, we study the existence and uniqueness of strong solution of a regularized model of the motion of a 3D nonlinear-viscous fluid with delay in the locally Lipschitz case, and further study the asymptotic behavior of solution.
\end{abstract}

\section{Subject Areas \\ Partial Differential Equation}

\section{Keywords}

Nonlinear-Viscous Fluid Equations, Existence and Uniqueness of Solution, Asymptotic Behaviour

\section{Problem Building}

In [1], the author introduced and studied the following regularized model of a nonlinear-viscous fluid motion:

$$
\frac{\partial u}{\partial t}+D_{\varepsilon}(u)-v \Delta u+B(u)+\nabla p=f(x, t), \quad \nabla \cdot u=0 .
$$

In many cases, we control the system by applying an external force [2]. This external force not only takes into account the current state of the system, but also considers the previous state, so the model can be better described [3] [4]. In this article we focus on the cases with time delay. Let $\Omega \in \mathbb{R}^{3}$ be a bounded open set with regular boundary $\Gamma$, then consider a regularized model of the motion of a nonlinear viscous on $\Omega$ with homogeneous Dirichlet boundary conditions. 


$$
\begin{cases}\frac{\partial u}{\partial t}+D_{\varepsilon}(u)-v \Delta u+B(u)+\nabla p=G(t, u(t-\rho(t))), & (0,+\infty) \times \Omega, \\ \nabla \cdot u=0, & (0,+\infty) \times \Omega, \\ u=0, & (0,+\infty) \times \Gamma, \\ u(\tau, x)=u^{0}(x), & x \in \Omega, \\ u(t, x)=\phi(t-\tau, x), & (\tau-h, \tau) \times \Omega .\end{cases}
$$

where $v>0$ is the viscosity coefficient and $\varepsilon$ is a positive constant. The unknown vector function $u(x, t)=\left(u_{1}(x, t), u_{2}(x, t), u_{3}(x, t)\right)$ represents the velocity of the fluid, $\tau \in \mathbb{R}$ is an initial time and $u^{0}$ indicates the initial velocity of the fluid. In addition, $G(t, u(t-\rho(t)))$ is an external force term that depends on $u(t-\rho(t))$, where $\rho(t) \geq 0$ is a delay function. When $h>0$ $\rho(t) \leq h$ are fixed, $\phi$ is a given velocity field defined at $(-h, 0)$. The function $p$ is the pressure and $(p, 1)_{H}=\int_{\Omega} p(x, t) \mathrm{d} x=0 . D_{\varepsilon}(u)$ is similar to the inertia term, denoted as

$$
D_{\varepsilon}(u)=\frac{1}{2 \varepsilon} \nabla\left[\ln \left(1+\varepsilon|u|^{2}\right)\right]-\frac{u \times(\nabla \times u)}{1+\varepsilon|u|^{2}} .
$$

here we denote by $B(u)=-\nabla \cdot\left(2 \mu\left(I_{2}(u)\right) E(u)\right)$, and the tensor $\varepsilon_{i j}$ is related to $u$, i.e.

$$
\varepsilon_{i j}(u)=\frac{1}{2}\left(\frac{\partial u_{i}}{\partial x_{j}}+\frac{\partial u_{j}}{\partial x_{i}}\right), \quad I_{2}^{2}(u)=E: E=\sum_{i, j}^{2} \varepsilon_{i j}^{2}(u) .
$$

Suppose $I_{2}(u)=s, \mu(s)$ satisfies the condition

1) $0<m_{1} \leq \mu(s) \leq m_{2}<\infty, \mu(s) \in C^{\infty}$. If $\mu^{\prime}(s)<0$, then $-s \mu^{\prime}(s) \leq \mu(s)$;

2) $s\left|\mu^{\prime}(s)\right| \leq \mu(s), \forall s \in(0,+\infty)$;

3) There is a positive constant $q$, such that $s\left|\mu^{\prime}(s)\right| \leq q \mu(s), \forall s \in(0,+\infty)$, $q<\frac{1}{2} \sqrt{\frac{m_{1}}{m_{2} \alpha}}$.

First, we introduce some basic knowledge of the Sobolev space in [5] [6]. Let $H=\left(L^{2}(\Omega)\right)^{3}, V=\left(H_{0}^{1}(\Omega)\right)^{3} . H^{\prime}$ and $V^{\prime}$ are the dual spaces of $H$ and $V$, so we obtain $V \subset H \equiv H^{\prime} \subset V^{\prime}$. Using $|\cdot|_{2}$ and $(\cdot, \cdot)$ to represent norm and inner product on $H$. We get $(u, v)=\int_{\Omega} u v \mathrm{~d} x, \forall u, v \in H$. Using $\|\cdot\|$ and $((\cdot, \cdot))$ to represent norm and inner product on $V$. We get $((u, v))=\int_{\Omega} \nabla u \cdot \nabla v \mathrm{~d} x, \forall u, v \in V . D(A)=V \cap\left(H^{2}(\Omega)\right)^{3}$ is the domain of $A$. For any $u \in D(A), A u=-P \Delta u$ is a Stokes operator.

$A \in \mathcal{L}\left(V, V^{\prime}\right) \cap \mathcal{L}\left(V \cap\left(H^{2}(\Omega)\right)^{3}, H\right)$ represents the continuous linear operator defined as follows: $\langle A u, \phi\rangle=(\nabla u, \nabla \phi), \forall u, \phi \in V$.

In addition, we assume $G: \mathbb{R} \times H \rightarrow H$ is such that

$\left(A_{1}\right)$ for any $u \in H, G(\cdot, u): \mathbb{R} \rightarrow H$ is measurable,

$\left(A_{2}\right)$ there exists a nonnegative function $g \in L_{\text {loc }}^{p}(\mathbb{R})$ for some $1<p<+\infty$, and a nondecreasing function $L:(0, \infty) \rightarrow(0, \infty)$, such that for all $R>0$, if 
$|u|_{2},|v|_{2} \leq R$, then $|G(t, u)|_{2}^{2}<g(t)|u|_{2}^{2}+f(t), \quad \forall t \in \mathbb{R}$,

$\left(A_{3}\right)$ there exists a nonnegative function $f \in L_{\text {loc }}^{1}(\mathbb{R})$, such that for any $u \in H$, we obtain $|G(t, u)|_{2}^{2}<g(t)|u|_{2}^{2}+f(t), \quad \forall t \in \mathbb{R}$.

Finally, we assume $\phi \in L^{2 p^{\prime}}(-h, 0 ; H), u^{0} \in H$, where $\frac{1}{p}+\frac{1}{p^{\prime}}=1$. In this case, we consider the delay function $\rho \in C^{1}(\mathbb{R})$, such that $0 \leq \rho(t) \leq h$, and there exists a constant $\rho_{*}$ satisfying $\rho^{\prime}(t) \leq \rho_{*}<1, \forall t \in \mathbb{R}$.

\section{Asymptotic Behavior of the Solution}

Proposition 2.1. [1]

$$
(B(u), u) \geq 2 \min _{s \geq 0} \mu(s)\|u\|^{2}=\beta_{1}\|u\|^{2},
$$

where $\beta_{1}=2 \min _{s \geq 0}|\mu(s)|$. And

$$
\begin{gathered}
(B(u)-B(v), u-v) \geq C\|u-v\|^{2}, \\
\left|\left(D_{\varepsilon}(u)-D_{\varepsilon}(v), u-v\right)\right| \leq \frac{2}{\sqrt{\varepsilon}}|u-v|_{2} \cdot\|u-v\|+\frac{3}{2}\|u\| \cdot\|u-v\|_{L^{4}(\Omega)}^{2} .
\end{gathered}
$$

It is easy to prove the following proposition by using the proof method of Theorem 4 in [7].

Proposition 2.2. For any $u \in D(A) \cap V$, there exists a constant $\beta_{2}>0$, such that

$$
(B(u), A u) \geq \beta_{2}|A u|_{2}^{2} .
$$

Definition 2.1. Let $\tau \in \mathbb{R}, u^{0} \in H$ and $L^{2 p^{\prime}}(-h, 0 ; H)$ be given. A weak solution of (2) is a function

$$
u \in L^{2 p^{\prime}}(-h, 0 ; H) \cap L^{2}(\tau, T ; V) \cap L^{\infty}(\tau, T ; H),
$$

for all $T>\tau$, such that

$$
\left\{\begin{array}{l}
\frac{\partial u}{\partial t}+D_{\varepsilon}(u)-v \Delta u+B(u)+\nabla p=G(t, u(t-\rho(t))), \\
u(\tau)=u^{0} \\
u(t)=\phi(t-\tau), \quad t \in(\tau-h, \tau) .
\end{array}\right.
$$

Remark: If $u$ is a weak solution of the (2) and we suppose $g(t)=g \circ \theta^{-1}(t)$, where $\theta:[\tau,+\infty] \rightarrow[\tau-\rho(\tau),+\infty)$ is differentiable and strictly increasing function given by $\theta(s)=s-\rho(s)$, we obtain

$$
\begin{aligned}
& \int_{\tau}^{T}|G(t, u(t-\rho(t)))|_{2}^{2} \mathrm{~d} s \\
& \leq \int_{\tau}^{T} g(t)|u(t-\rho(t))|_{2}^{2} \mathrm{~d} t+\int_{\tau}^{T} f(t) \mathrm{d} t \\
& \leq \frac{1}{1-\rho_{*}} \int_{\tau-\rho(\tau)}^{T-\rho T} \tilde{g}(t)|u(t)|_{2}^{2} \mathrm{~d} t+\int_{\tau}^{T} f(t) \mathrm{d} t \\
& \leq \frac{1}{1-\rho_{*}} \int_{\tau-\rho(\tau)}^{T} \tilde{g}(t)|u(t)|_{2}^{2} \mathrm{~d} t+\int_{\tau}^{T} f(t) \mathrm{d} t,
\end{aligned}
$$


So for all $T>\tau$, taking $\tilde{g} \in L^{p}(\tau-\rho(\tau), T)$, we get $G(t, u(t-\rho(t))) \in L^{2}(\tau, T ; H)$. Obviously, it is easy to prove a $\frac{\mathrm{d}}{\mathrm{d} t} \in L^{2}\left(\tau, T ; V^{\prime}\right)$, $u \in C([\tau,+\infty) ; H)$, and satisfies the energy equality, for all $\tau<s<t$,

$$
|u(t)|_{2}^{2}-|u(s)|_{2}^{2}+2\left(v+\beta_{1}\right) \int_{s}^{t} \mid\|(r)\|^{2} \mathrm{~d} r=2 \int_{s}^{t}(G(r, u(r-\rho(r))), u(r)) \mathrm{d} r .
$$

Theorem 2.1. In the case of satisfying $\left(A_{1}\right)-\left(A_{3}\right)$, assume that $\tau \in \mathbb{R}, u^{0} \in H$ and $\phi \in L^{2 p^{\prime}}(-h, 0 ; H)$ are given. We get

1) there exists a unique weak solution $u$ of (2) which is, in fact, a strong solution in the sense that

$$
u \in C([\tau+\eta, T] ; V) \cap L^{2}(\tau+\eta, T ; D(A)), \quad \forall T-\tau>\eta>0 .
$$

2) if $u^{0} \in V$, we obtain

$$
u \in C([\tau, T] ; V) \cap L^{2}(\tau, T ; D(A)), \quad \forall T>\tau .
$$

Proof: It is similar to the proof method of Theorem 3.1 in [8]. Combining Proposition 2.1 and Proposition 2.2, the Galerkin method can be used to prove the existence and uniqueness of the solution in (2). Since this method is standard, it is omitted here.

Theorem 2.2. In the case where $g \in L^{\infty}(\mathbb{R})$ satisfies $\left(A_{1}\right)-\left(A_{3}\right)$, we assume

$$
\lambda_{1}^{2}\left(v+\beta_{1}\right)^{2}\left(1-\rho_{*}\right)>|g|_{\infty},
$$

where $|g|_{\infty}=\|g\|_{L^{\infty}(\mathbb{R})}$. When $\eta>0$, we define a unique solution that satisfies

$$
\eta-\lambda_{1}\left(v+\beta_{1}\right)+\frac{|g|_{\infty} \mathrm{e}^{\eta h}}{\lambda_{1}\left(v+\beta_{1}\right)\left(1-\rho_{*}\right)}=0 .
$$

for any $\left(u^{0}, \phi\right) \in H \times L^{2}(-h, 0 ; H)$ and $\tau \in \mathbb{R}$. The solution is $u\left(t ; \tau, u^{0}, \phi\right)$ of (2) that satisfies

$$
\begin{aligned}
\left|u\left(t ; \tau, u^{0}, \phi\right)\right|_{2}^{2} \leq & \left(\left|u^{0}\right|_{2}^{2}+\frac{|g|_{\infty} \mathrm{e}^{\eta h}}{\lambda_{1}\left(v+\beta_{1}\right)\left(1-\rho_{*}\right)} \int_{-h}^{0} \mathrm{e}^{\eta s}|\phi(s)|_{2}^{2} \mathrm{~d} s\right) \mathrm{e}^{\eta(\tau-t)} \\
& +\frac{\mathrm{e}^{-\eta t}}{\lambda_{1}\left(v+\beta_{1}\right)} \int_{\tau}^{t} \mathrm{e}^{\eta s} f(s) \mathrm{d} s, \quad \forall t \geq \tau .
\end{aligned}
$$

In particular, if $\int_{\tau}^{\infty} \mathrm{e}^{\eta s} f(s) \mathrm{d} s<\infty$, we get $u\left(t ; \tau, u^{0}, \phi\right) \rightarrow 0$ when $t \rightarrow+\infty$.

Proof: We denote $u(t)=u\left(t ; \tau, u^{0}, \phi\right)$ is the solution of (2), where the initial values are $\tau, u^{0}$ and $\phi$. Multiplying (2) by $u$, and integrating it on $\Omega$, it yields

$$
\frac{1}{2} \frac{\mathrm{d}}{\mathrm{d} t}|u|_{2}^{2}+\left(v+\beta_{1}\right) \|\left. u\right|^{2}=(G(t, u(t-\rho(t))), u)
$$

So

$$
\frac{\mathrm{d}}{\mathrm{d} t}\left(\mathrm{e}^{\eta t}|u|_{2}^{2}\right)=\eta \mathrm{e}^{\eta t}|u|_{2}^{2}-2\left(v+\beta_{1}\right) \mathrm{e}^{\eta t}\|u(t)\|^{2}+2 \mathrm{e}^{\eta t}(G(t, u(t-\rho(t))), u) .
$$

Using Young inequality and Pacaré inequality, we obtain 


$$
\frac{\mathrm{d}}{\mathrm{d} t}\left(\mathrm{e}^{\eta t}|u|_{2}^{2}\right)=\left[\eta-\lambda_{1}\left(v+\beta_{1}\right)\right] \mathrm{e}^{\eta t}|u|_{2}^{2}+\frac{1}{\lambda_{1}\left(v+\beta_{1}\right)} \mathrm{e}^{\eta t}|G(t, u(t-\rho(t)))|_{2}^{2} .
$$

for all $t \geq \tau$, we have

$$
\int_{\tau}^{t} \mathrm{e}^{\eta s}|G(t, u(t-\rho(t)))|_{2}^{2} \mathrm{~d} s \leq|g|_{\infty} \int_{\tau}^{t} \mathrm{e}^{\eta s}|u(s-\rho(s))|_{2}^{2} \mathrm{~d} s+\int_{\tau}^{t} \mathrm{e}^{\eta s} f(s) \mathrm{d} s .
$$

and

$$
\begin{aligned}
& \int_{\tau}^{t} \mathrm{e}^{\eta s}|u(s-\rho(s))|_{2}^{2} \mathrm{~d} s \\
& \leq \frac{\mathrm{e}^{\eta h}}{1-\rho_{*}} \int_{\tau}^{t} \mathrm{e}^{\eta s}|u(s)|_{2}^{2} \mathrm{~d} s+\frac{\mathrm{e}^{\eta h}}{1-\rho_{*}} \int_{\tau-h}^{\tau} \mathrm{e}^{\eta s}|\phi(s-\tau)|_{2}^{2} \mathrm{~d} s \\
& =\frac{\mathrm{e}^{\eta h}}{1-\rho_{*}} \int_{\tau}^{h} \mathrm{e}^{\eta s}|u(s)|_{2}^{2} \mathrm{~d} s+\frac{\mathrm{e}^{\eta h}}{1-\rho_{*}} \int_{-h}^{0} \mathrm{e}^{\eta s}|\phi(s)|_{2}^{2} \mathrm{~d} s .
\end{aligned}
$$

Therefore, integrating (5) on $[\tau, t]$, we get

$$
\begin{aligned}
& \mathrm{e}^{\eta t}|u(t)|_{2}^{2}-\mathrm{e}^{\eta \tau}|u(\tau)|_{2}^{2} \\
& \leq\left[\eta-\lambda_{1}\left(v+\beta_{1}\right)\right] \int_{\tau}^{t} \mathrm{e}^{\eta s}|u(s)|_{2}^{2} \mathrm{~d} s+\int_{\tau}^{t} \mathrm{e}^{\eta s} f(s) \mathrm{d} s \\
& \quad+\frac{1}{\lambda_{1}\left(v+\beta_{1}\right)}|g|_{\infty}\left(\frac{\mathrm{e}^{\eta h}}{1-\rho_{*}} \int_{\tau}^{h} \mathrm{e}^{\eta s}|u(s)|_{2}^{2} \mathrm{~d} s+\frac{\mathrm{e}^{\eta(h+\tau)}}{1-\rho_{*}} \int_{-h}^{0} \mathrm{e}^{\eta s}|\phi(s)|_{2}^{2} \mathrm{~d} s\right) .
\end{aligned}
$$

Combining (3), for all $t \geq \tau$, we obtain

$$
\begin{aligned}
\left|u\left(t ; \tau, u^{0}, \phi\right)\right|_{2}^{2} \leq & \left(\left|u^{0}\right|_{2}^{2}+\frac{|g|_{\infty} \mathrm{e}^{\eta h}}{\lambda_{1}\left(v+\beta_{1}\right)\left(1-\rho_{*}\right)} \int_{-h}^{0} \mathrm{e}^{\eta s}|\phi(s)|_{2}^{2} \mathrm{~d} s\right) \mathrm{e}^{\eta(\tau-t)} \\
& +\frac{\mathrm{e}^{-\eta t}}{\lambda_{1}\left(v+\beta_{1}\right)} \int_{\tau}^{t} \mathrm{e}^{\eta s} f(s) \mathrm{d} s .
\end{aligned}
$$

Theorem 2.3. In the case where $g \in L^{\infty}(\mathbb{R})$ satisfies $\left(A_{1}\right)-\left(A_{3}\right)$, we assume $\lambda_{1}^{2}\left(v+\beta_{2}\right)^{2}\left(1-\rho_{*}\right)>|g|_{\infty}$,

where $|g|_{\infty}=\|g\|_{L^{\infty}(\mathbb{R})}$. When $\eta>0$, we define a unique solution that satisfies

$$
\eta+\frac{1}{2 \varepsilon\left(v+\beta_{2}\right)}-\lambda_{1}\left(v+\beta_{2}\right)+\frac{|g|_{\infty} \mathrm{e}^{\eta h}}{\lambda_{1}\left(v+\beta_{2}\right)\left(1-\rho_{*}\right)}=0 .
$$

for any $\left(u^{0}, \phi\right) \in V \times L^{2}(-h, 0 ; V)$ and $\tau \in \mathbb{R}$. The solution $u\left(t ; \tau, u^{0}, \phi\right)$ of $(2)$ satisfies

$$
\begin{aligned}
\left\|u\left(t ; \tau, u^{0}, \phi\right)\right\|^{2} \leq & \left(\left\|u^{0}\right\|^{2}+\frac{|g|_{\infty} \mathrm{e}^{\eta h}}{\lambda_{1}\left(v+\beta_{2}\right)\left(1-\rho_{*}\right)} \int_{-h}^{0} \mathrm{e}^{\eta s}|\phi(s)|_{2}^{2} \mathrm{~d} s\right) \mathrm{e}^{\eta(\tau-t)} \\
& +\frac{\mathrm{e}^{-\eta t}}{\lambda_{1}\left(v+\beta_{2}\right)} \int_{\tau}^{t} \mathrm{e}^{\eta s} f(s) \mathrm{d} s, \quad \forall t \geq \tau .
\end{aligned}
$$

In particular, if $\int_{\tau}^{\infty} \mathrm{e}^{\eta s} f(s) \mathrm{d} s<\infty$, we have $u\left(t ; \tau, u^{0}, \phi\right) \rightarrow 0$ when $t \rightarrow+\infty$.

Proof: We denote $u(t)=u\left(t ; \tau, u^{0}, \phi\right)$ is the solution of (2), where the initial values are $\tau, u^{0}$ and $\phi$. Multiplying (2) by $A u$, and integrating it on $\Omega$, it yields 


$$
\frac{1}{2} \frac{\mathrm{d}}{\mathrm{d} t}\|u\|^{2}+v|A u|_{2}^{2}+(B(u), A u)+\left(D_{\varepsilon}(u), A u\right)=(G(t, u(t-\rho(t))), A u) .
$$

Due to

$$
\begin{aligned}
\left(D_{\varepsilon}(u), A u\right) & =\int_{\Omega} \frac{u \cdot \nabla u}{1+\varepsilon|u|^{2}} A u \mathrm{~d} x \leq \int_{\Omega} \frac{1}{2 \sqrt{\varepsilon}}|\nabla u \cdot A u| \mathrm{d} x \\
& \leq \frac{1}{2 \sqrt{\varepsilon}}|\nabla u|_{2}|A u|_{2} \leq\left.\frac{1}{4 \varepsilon\left(v+\beta_{2}\right)}\left|u \|^{2}+\frac{v+\beta_{2}}{4}\right| A u\right|_{2} ^{2} .
\end{aligned}
$$

Combining Proposition 2.2, we get

$$
\begin{aligned}
& \frac{\mathrm{d}}{\mathrm{d} t}\left(\mathrm{e}^{\eta t}\|u\|^{2}\right)+\frac{3}{2}\left(v+\beta_{2}\right) \mathrm{e}^{\eta t}|A u|_{2}^{2} \\
& \leq\left[\eta+\frac{1}{2 \varepsilon\left(v+\beta_{2}\right)}\right] \mathrm{e}^{\eta t}\|u\|^{2}+2 \mathrm{e}^{\eta t}(G(t, u(t-\rho(t))), A u) .
\end{aligned}
$$

According to Young inequality and Pacaré inequality, we obtain

$$
\begin{aligned}
\frac{\mathrm{d}}{\mathrm{d} t}\left(\mathrm{e}^{\eta t}\|u\|^{2}\right) \leq & {\left[\eta+\frac{1}{2 \varepsilon\left(v+\beta_{2}\right)}-\lambda_{1}\left(v+\beta_{2}\right)\right] \mathrm{e}^{\eta t}\|u\|^{2} } \\
& +\frac{1}{\lambda_{1}\left(v+\beta_{2}\right)}\left|\mathrm{e}^{\eta t} G(t, u(t-\rho(t)))\right|_{2}^{2} .
\end{aligned}
$$

Therefore, combining (6) and (7), integrating (10) on $[\tau, t]$, we get

$$
\begin{aligned}
& \mathrm{e}^{\eta t}\|u(t)\|^{2}-\mathrm{e}^{\eta \tau}\|u(\tau)\|^{2} \\
& \leq\left[\eta+\frac{1}{2 \varepsilon\left(v+\beta_{2}\right)}-\lambda_{1}\left(v+\beta_{2}\right)\right] \int_{\tau}^{t} \mathrm{e}^{\eta s}\|u(s)\|^{2} \mathrm{~d} s+\frac{1}{\lambda_{1}\left(v+\beta_{2}\right)} \int_{\tau}^{t} \mathrm{e}^{\eta s} f(s) \mathrm{d} s \\
& +\frac{1}{\lambda_{1}\left(v+\beta_{2}\right)}|g|_{\infty}\left(\frac{\mathrm{e}^{\eta h}}{1-\rho_{*}} \int_{\tau}^{h} \mathrm{e}^{\eta s}\|u(s)\|^{2} \mathrm{~d} s+\frac{\mathrm{e}^{\eta(h+\tau)}}{1-\rho_{*}} \int_{-h}^{0} \mathrm{e}^{\eta s}|\phi(s)|_{2}^{2} \mathrm{~d} s\right) .
\end{aligned}
$$

from (8), for all $t \geq \tau$, we obtain

$$
\begin{aligned}
& \left\|u\left(t ; \tau, u^{0}, \phi\right)\right\|^{2} \\
& \leq\left(\left\|u^{0}\right\|^{2}+\frac{|g|_{\infty} \mathrm{e}^{\eta h}}{\lambda_{1}\left(v+\beta_{2}\right)\left(1-\rho_{*}\right)} \int_{-h}^{0} \mathrm{e}^{\eta s}|\phi(s)|_{2}^{2} \mathrm{~d} s\right) \mathrm{e}^{\eta(\tau-t)} \\
& \quad+\frac{\mathrm{e}^{-\eta t}}{\lambda_{1}\left(v+\beta_{2}\right)} \int_{\tau}^{t} \mathrm{e}^{\eta s} f(s) \mathrm{d} s .
\end{aligned}
$$

\section{Funding}

This work was supported by the National Natural Sciences Foundation of China (No. 11571283).

\section{Conflicts of Interest}

The authors declare no conflicts of interest regarding the publication of this paper. 


\section{References}

[1] Agranovich, Y.Y. and Zvyagin, V.G. (2002) Investigations of Properties of Attractors for a Regularized Model of the Motion of a Nonlinear-Viscous Fluid. Theory Algebra, 2, 50-58.

[2] Temam, R. (1968) Une méthode d'approximation de la solution des équations de Navier-Stokes. Bulletin de la Société Mathématique de France, 96, 115-152. https://doi.org/10.24033/bsmf.1662

[3] Caraballo, T., Márquez-Durán, A.M. and Real, J. (2009) Asymptotic Behaviour of the Three-Dimensional $\alpha$-Navier-Stokes Model with Locally Lipschitz Delay Forcing Terms. Nonlinear Analysis Theory Methods and Applications, 71, e271-e282. https://doi.org/10.1016/j.na.2008.10.048

[4] Sobolevskii, P.E. (1985) The Existence of Solutions of a Mathematical Model of a Nonlinear Viscous Fluid. Doklady Akademii Nauk SSSR, 285, 44-48.

[5] Temam, R. (1984) Navier-Stokes Equations: Theory and Numerical Analysis. North-Holland Pub. Co., New York.

[6] Temam, R. (1998) Infinite-Dimensional Dynamical Systems in Mechanics and Physics. 2nd Edition, Springer-Verlag, New York.

[7] Agranovich, Y.Y. and Khatskevich, V.L. (2012) Mathematical Modeling of Nonlinearly Viscous Fluid Motion: Strong Solutions. Automation and Remote Control, 73, 171-180. https://doi.org/10.1134/s0005117912010158

[8] Caraballo, T., Real, J., et al. (2014) Three-Dimensional System of Globally Modified Navier-Stokes Equations with Delay. International Journal of Bifurcation and Chaos, 20, 1-8. https://doi.org/10.1142/s0218127410027428 